\title{
Local feature extraction based facial emotion recognition: A survey
}

\author{
Khadija Slimani $^{1}$, Mohamed Kas ${ }^{2}$, Youssef El merabet ${ }^{3}$, Yassine Ruichek ${ }^{4}$, Rochdi Messoussi ${ }^{5}$ \\ ${ }^{1,2,3,5}$ Laboratory Systems of Telecommunication and Decision Engineering (LASTID), Faculty of Sciences, \\ Ibn Tofail University, Morocco \\ ${ }^{2,4}$ CIAD UMR 7533, Université Bourgogne Franche-Comté, UTBM, France
}

\section{Article Info \\ Article history: \\ Received Oct 2, 2019 \\ Revised Feb 25, 2020 \\ Accepted Mar 4, 2020}

\section{Keywords:}

Basic emotion

Features extraction

Image processing

Machine learning

\begin{abstract}
Notwithstanding the recent technological advancement, the identification of facial and emotional expressions is still one of the greatest challenges scientists have ever faced. Generally, the human face is identified as a composition made up of textures arranged in micro-patterns. Currently, there has been a tremendous increase in the use of Local Binary Pattern based texture algorithms which have invariably been identified to being essential in the completion of a variety of tasks and in the extraction of essential attributes from an image. Over the years, lots of LBP variants have been literally reviewed. However, what is left is a thorough and comprehensive analysis of their independent performance. This research work aims at filling this gap by performing a large-scale performance evaluation of 46 recent state-of-the-art LBP variants for facial expression recognition. Extensive experimental results on the well-known challenging and benchmark KDEF, JAFFE, CK and MUG databases taken under different facial expression conditions, indicate that a number of evaluated state-of-the-art LBP-like methods achieve promising results, which are better or competitive than several recent state-of-the-art facial recognition systems. Recognition rates of 100\%, 98.57\%, 95.92\% and $100 \%$ have been reached for CK, JAFFE, KDEF and MUG databases, respectively.
\end{abstract}

Copyright $\left({ }_{0} 2020\right.$ Insitute of Advanced Engineeering and Science. All rights reserved.

\section{Corresponding Author:}

Slimani Khadija,

Laboratory Systems of Telecommunication and Decision Engineering (LASTID), Faculty of Sciences, Ibn Tofail University, Kenitra, Morocco.

Email: slimani.khadija@uit.ac.ma

\section{INTRODUCTION}

With the development of artificial intelligence and pattern recognition, computer based facial expression recognition has attracted many researchers in the domain of computer vision. Several studies have shown that the facial expression contributes to better understand the conversations $[1,2]$, and it helps to express the individual's internal emotions, also, it is considered as the main modality for human communication.

Recent progresses in psychology and neuroscience fields give a more positive interpretation of the emotions role in human behavior [3]. The facial emotion recognition system resides of three important steps; face detection, feature extraction and classification. By taking image or series of images as input, the most important step is feature extraction that allows to describe the input images and calculate their characteristic vector using a given operator. Indeed, extracting poor features involves producing poor recognition quality even with the use of best classifiers. Because of the exceptional exhibition of LBP based techniques, they have developed as one of the most unmistakable local image descriptors. Although initially intended for texture analysis [4], the LBP descriptor has given excellent outcomes in different applications because of its invariance to monotonic global graylevel changes, furthermore, its better resistance against brightening changes property in real-world 
applications including face recognition. Another equally important property is its computational effortlessness and the low length of its histogram vector, which make it ready to examine images in challenging real-time settings. The achievement of the LBP in numerous applications conceived an offspring of an immense number of LBP variations, which have been proposed and keep on being proposed. Without a doubt, since Ojala's work [4] and because of its adaptability and effectiveness, the general LBP-like way of thinking has demonstrated extremely well known, and an extraordinary assortment of LBP variations have been proposed in the writing to improve discriminative power, robustness, and appropriateness of LBP. The main objective of this study is to perform a large scale performance evaluation for facial emotion recognition, assessing 46 recent state-of-theart texture features, on four widely-used benchmark databases. Performance of the adopted facial expression recognition system coupled with the best evaluated texture descriptor on each dataset is compared against those of state-of-the-art approaches. We disclose in the experimental section the fact that some descriptors originally proposed for applications other than facial emotional recognition allow outperforming several recent state-ofthe-art systems. The remaining sections of this research work are arranged in the following way: Section 2. reviews the traditional LBP operator as well as some of its recent and popular variants. Section 3. reviews the few existing surveys on texture descriptor based classification and recognition as well as the evaluated stateof-the art LBP-like methods. Section 4. provides detailed explanation on the results of the experiments while comparing the performances of the best performing descriptors on each tested datasets with those of recent state-of-the-art facial emotional recognition systems. Finally, section 5. draw this paper to a close by proposing some future research perspectives.

\section{BRIEF REVIEW OF EXISTING METHODS}

The original Local Binary Pattern (LBP) operator proposed by Ojala et al [4], which consists in coding the pixel-wise information in an image, is a powerful texture analysis descriptor. It aims to search micro-textons in local regions. The value $\mathbf{I}_{p}$ of the pixels in a $3 \times 3$ grayscale image patch around the central pixel $\mathbf{I}_{c}$ are turned into binary values ( 0 or 1$)$ by comparing them with $\mathbf{I}_{c}$ (value of the central pixel). The obtained binary numbers are encoded to characterize a local structure pattern and then the code is transformed into decimal number. Once a LBP code of each pixel is obtained, a histogram is built to represent the texture image. For a $3 \times 3$ neighborhood, the definition of the kernel function of LBP operator is given in (cf. Eq (1)), where $\mathbf{I}_{p}(\mathrm{p} \in\{1,2, \ldots, \mathrm{P}\})$ signifies the gray levels of the peripheral pixels, $\mathrm{P}$ corresponds to the number of neighboring pixels $(\mathrm{P}=8)$ and $\varphi(\cdot)$ is the Heaviside step function (cf. Eq (1)).

$$
\mathbf{L B P}\left(\mathbf{I}_{\mathbf{c}}\right)=\sum_{\mathbf{p}=\mathbf{1}}^{\mathbf{P}=\mathbf{8}} \varphi\left(\mathbf{I}_{\mathbf{p}}-\mathbf{I}_{\mathbf{c}}\right) \times \mathbf{2}^{\mathbf{p}-\mathbf{1}}, \quad \varphi(\mathbf{z})=\left\{\begin{array}{l}
1, \mathbf{z} \geq 0 \\
0, \mathbf{z}<0
\end{array}\right.
$$

Local binary patterns by neighborhoods $(n L B P d)$ operator [5] consists in encoding the relationship between each pair of the peripheral pixels $\mathbf{I}_{0}, \mathbf{I}_{1}, \mathbf{I}_{2}, \ldots, \mathbf{I}_{7}$ around the central pixel $\mathbf{I}_{c}$ in a $3 \times 3$ square neighborhood. The pairs of pixels are compared with sequential neighbors or within neighbors possesing a distance length $\mathrm{d}$. The kernel function of $n L B P d$ code is defined by (cf. Eq. (2)). When $\mathrm{d}=1$, the binary code of the central pixel $\mathbf{I}_{c}$ is gotten as below (Eq. (3)):

$$
\operatorname{nLBP}_{d}\left(\mathbf{I}_{\mathbf{c}}\right)=\sum_{p=0}^{P-1} \varphi\left(\mathbf{I}_{\mathbf{p}}, \mathbf{I}_{(\mathbf{p}+\mathbf{d} \bmod \mathrm{P})}\right) \times 2^{p}
$$

$$
\mathbf{I}_{c}=\varphi\left(\mathbf{I}_{0}>\mathbf{I}_{1}\right), \varphi\left(\mathbf{I}_{1}>\mathbf{I}_{2}\right), \varphi\left(\mathbf{I}_{2}>\mathbf{I}_{3}\right), \varphi\left(\mathbf{I}_{3}>\mathbf{I}_{4}\right), \varphi\left(\mathbf{I}_{4}>\mathbf{I}_{5}\right), \varphi\left(\mathbf{I}_{5}>\mathbf{I}_{6}\right), \varphi\left(\mathbf{I}_{6}>\mathbf{I}_{7}\right), \varphi\left(\mathbf{I}_{7}>\mathbf{I}_{8}\right)
$$

The procedure of Local Graph Structure (LGS) descriptor introduced by Abusham et al. [6] is to exploit the dominant graph process in order to encode the spatial data for any pixel in the image. LGS is based on local graph structures in local graph neighborhood. The graph structure of LGS represents more left-handed neighbor pixels than right-handed ones. To overcome this defect, Extended Local Graph Structure (ELGS) operator is proposed [7]. The procedure for ELGS is based on using the LGS texture descriptor to build two descriptions (horizontally and vertically) and then combine them into a global description. 


\section{EVALUATED STATE-OF-THE-ART LBP VARIANTS}

The pioneering LBP work [4] and its success in numerous computer vision problems and applications has inspired the development of great number of new powerful LBP variants. LBP descriptor is adaptable to suit in many different applications requirements. Indeed, after Ojala's work, e.g., Heikkila et al [8], several modifications and extensions of LBP have been developed with the aim to increase its robustness and discriminative power. These extensions and modifications of LBP, developed usually in conjunction with their intended applications (see Table 1), focus on several aspects of the LBP method such as, Quantization to multiple level via thresholding; sampeling local feature vectors and pixel patterns with some neighborhood topology; combining multiple complementary features within LBP-like and with non-LBP descriptors for both images and videos and finally, regrouping and merging patterns to increase distinctiveness.

Table 1. Summary of texture descriptors tested.

\begin{tabular}{|c|c|c|c|c|}
\hline Ref & Year & Complete name & Abbreviation & Application \\
\hline [4] & 2002 & Local Binary Pattern & LBP & Texture classification \\
\hline [9] & 2003 & Simplified Texture Unit + & STU+ & Texture classification \\
\hline [10] & 2004 & Gradient texture unit coding & GTUC & Texture classification \\
\hline [11] & 2005 & Difference Symmetric Local Graph Structure & DSLGS & Finger vein recognition \\
\hline [8] & 2006 & Center-Symmetric Local Binary Patterns & CSLBP & Texture classification \\
\hline [12] & 2008 & Centralized Binary Pattern & CBP & Facial expression recognition \\
\hline [13] & 2010 & Local Ternary Patterns & LTP & Face recognition \\
\hline [14] & 2010 & Directional Binary Code & $\mathrm{DBC}$ & Face recognition \\
\hline [15] & 2010 & Improved Local Ternary Patterns & ILTP & Medical image analysis \\
\hline [16] & 2010 & Local Directional Pattern & LDP & Face recognition \\
\hline [17] & 2011 & Binary Gradient Contours (1) & BGC1 & Texture classification \\
\hline [17] & 2011 & Binary Gradient Contours (2) & BGC2 & Texture classification \\
\hline [17] & 2011 & Binary Gradient Contours (3) & BGC3 & Texture classification \\
\hline [18] & 2011 & Center-Symmetric Local Ternary Patterns & CSLTP & Feature description \\
\hline$[18]$ & 2011 & Extended Center-Symmetric Local Ternary Patterns & eCSLTP & Image retrieval \\
\hline [19] & 2011 & Improved Local Binary Patterns & ILBP & Face detection \\
\hline$[6]$ & 2011 & Local Graph Structure & LGS & Face recognition \\
\hline [20] & 2012 & Local Maximum Edge Binary Patterns & LMEBP & Image retrieval \\
\hline [16] & 2013 & Improved binary gradient contours (1) & IBGC1 & Texture classification \\
\hline [21] & 2013 & Local Directional Number Pattern & LDN & Face expression analysis \\
\hline$[22]$ & 2013 & Local Gray Code Pattern & LGCP & Face expression analysis \\
\hline [23] & 2013 & Rotated Local Binary Pattern & RLBP & Texture classification \\
\hline [24] & 2015 & Adaptive Extended Local Ternary Pattern & AELTP & Texture classification \\
\hline$[5]$ & 2015 & Directional Local Binary Patterns & $\mathrm{dLBP} \alpha$ & Texture classification \\
\hline [5] & 2015 & Local Binary Patterns by neighborhoods & $\mathrm{nLBPd}$ & Texture classification \\
\hline [25] & 2015 & Maximum Edge Position Octal Pattern & MMEPOP & Image retrieval \\
\hline [26] & 2015 & Multi-Orientation Weighted Symmetric Local Graph Structure & MOW-SLGS & Finger vein recognition \\
\hline [27] & 2015 & Orthogonal Symmetric Local Ternary Pattern & OSLTP & Image region description \\
\hline [26] & 2015 & Symmetric Local Graph Structure & SLGS & Finger vein recognition \\
\hline [28] & 2015 & eXtended Center-Symmetric Local Binary Pattern & XCS_LBP & Texture classification \\
\hline [29] & 2016 & Adaptive Local Ternary Patterns & ALTP & Face recognition \\
\hline [29] & 2016 & Center-Symmetric ALTP & CSALTP & Face recognition \\
\hline [30] & 2016 & Diagonal Direction Binary Pattern & DDBP & Face recognition \\
\hline [7] & 2016 & Extended Local Graph Structure & ELGS & Texture classification \\
\hline [31] & 2016 & Local Extreme Sign Trio Patterns & LESTP & Image retrieval \\
\hline [32] & 2016 & Quad Binary Pattern & QBP & Target tracking \\
\hline [31] & 2016 & Sign Maximum Edge Position Octal Pattern & SMEPOP & Image retrieval \\
\hline [33] & 2016 & Complete Eight Local Directional Patterns & CELDP & Face recognition \\
\hline [34] & 2017 & Centre Symmetric Quadruple Pattern & CSQP & Facial image recognition and retrieval \\
\hline [35] & 2017 & Local Directional Binary Patterns & LDBP & Texture classification \\
\hline [36] & 2017 & Local neighborhood difference pattern & LNDP & Natural and texture image retrieval \\
\hline [37] & 2017 & Local Quadruple Pattern & LQPAT & Facial image recognition and retrieval \\
\hline [38] & 2018 & Local Diagonal Extrema Number Pattern & LDENP & Face recognition \\
\hline [39] & 2018 & Local Concave-and-Convex Micro-Structure Patterns & LCCMSP & Texture classification \\
\hline [40] & 2018 & Local Directional Ternary Pattern & LDTP & Texture classification \\
\hline [41] & 2018 & Repulsive-and-Attractive Local Binary Gradient Contours & RALBGC & Texture classification \\
\hline
\end{tabular}


There are several researches reported in the literature that are devoted to surveying LBP and its variants. One can cite:

(a) Hadid et al. [42] reviewed 13 LBP variants and provided a comparative analysis on two different problems which are gender and texture classification.

(b) The work of Fernandez et al. [43] attempted to build a general framework for texture examination that the authors refer to as histograms of equivalent patterns (HEP). A set of 38 LBP variants and non LBP strategies are executed and experimentally assessed on eleven texture datasets.

(c) Huang et al. [44] displayed a survey of LBP variants in the application region of facial image processing. However, there is no experimental study of the LBP strategies themselves.

(d) Nanni et al. [45] examined the performance of LBP based texture descriptors in a fairly specific and narrow application, which consists in classifying cell and tissue images of five datasets.

(e) Michael Bereta et al. [46] highlighted many types of local descriptors including local binary patterns and their combination with Gabor filters. They examined only 14 LBP variants on FERET database.

(f) Lumini et al. [47] evaluated the effectiveness of LBP, HOG, POEM, MBC, HASC, GOLD, RICLBP, and CLBP descriptors. Each of these feature extraction methods is carried out only on two datasets: FERET and the Labeled Faces in the Wild (LFW).

(g) Liu et al. [48] provided a systematic review of LBP variants while regrouping them into different categories. 40 texture features including thirty two LBP-like descriptors and eight non-LBP methods are evaluated and compared on thirteen texture datasets.

(h) Slimani et al. [49] reviewed the performance of 22 state-of-the-art LBP-like descriptors and some of its recent variations and provides a comparative analysis on facial expression recognition problem using two benchmark databases.

It can be inferred that there is a limited number of state-of-the-art published works which are devoted to survey LBP-like methods in texture and face recognition and in particular facial emotion recognition which is practically nonexistent. Note that, most of these works remain limited in terms of number of LBP-like descriptors reviewed and tested datasets, suffer from lack of recent LBP variants and some of them do not include experimental evaluation. Since no broad assessment has been performed on an incredible number of LBP variations, and considering recent rapid increase in the number of publications on LBP-like descriptors, this paper aims to provide such a comparative study in facial emotion recognition problem and offers a more up-to-date introduction to the area. For that, 46 recent state-of-the-art LBP variants are evaluated and compared over four challenging representative widely-used facial expression databases. The performance of the best texture descriptor on each dataset is also composed to those of state-of-the-art facial emotion recognition systems. Note that for the descriptors, we utilized the original source code if it is freely accessible; otherwise we have built up our own implementation. The evaluated state-of-the-art texture descriptors and their intended applications are summarized in Table 1.

\section{EXPERIMENTAL RESULTS AND DISCUSSION}

In this section, the state-of-the-art LBP variants summarized in Table 1 are extensively evaluated and compared over four publicly available facial expression datasets (see section 4.2.). In addition, performance of the best performing method on each dataset has been compared against those of recent state-of-the-art facial emotion recognition systems. The following subsections describe: 1) the experimental configuration; 2) the datasets considered in the experiments, 3) the obtained results and 4) comparisons with other existing approaches.

\subsection{Experimental configuration}

In order to systematically evaluate the performance of the tested methods, we setup a comparative analysis through a supervised image classification task. Similar to most state-of-the-art facial expression recognition systems, the adopted system, shown in Figure 1, involves several steps including 1) image processing to alter and resize faces to have a common resolution; 2) feature extraction using the evaluated LBP variants; 3) histogram vector calculation. In this step, in order to incorporate more spatial information into the final feature vectors, the obtained feature images were spatially divided into multiple non-overlapping regions and histograms were extracted from each region. For example, the LBP code map is divided into $\mathrm{m} \times \mathrm{n}$ nonoverlapping sub-regions, from each of which a sub-histogram feature is extracted and is normalized to sum 
one. By concatenating these regional sub-histograms into a single vector, a final LBP based facial emotion representation is obtained; and 4) image classification using the SVM classifier. In this step, the images of each dataset are preliminarily divided into a random split containing two sub-sets, one for the training and the other for testing. In the experiments, we tackled the 7-expression classification problems and overall results are computed as the average of the per-class accuracies and not the average accuracy of all samples, which avoids biasing toward expressions with more samples in the databases.

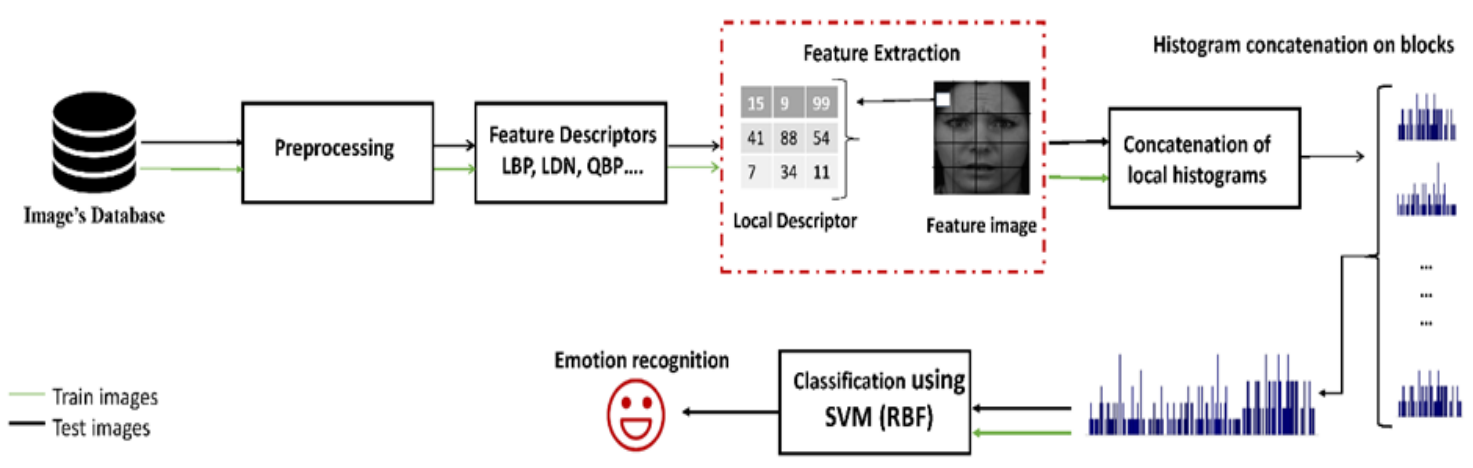

Figure 1. Outline of the adopted facial emotion recognition system.

\subsection{Tested datasets}

In our experiments, we used four benchmark databases; the Cohn Kanade (CK), the Japanese Female Facial Expression (JAFFE), the Karolinska Directed Emotional Faces (KDEF) and the Multimedia Understanding Group (MUG) databases. The main characteristics of each database are described herein below. The four datasets include facial expressions of six basic emotions; Anger, Disgust, Fear, Happiness, Sadness, Surprise and the neutral facial expression.

(a) The JAFFE database [50] contains 213 facial expression images from 10 Japanese females where every subject expresses three times the seven facial expressions. The images have a resolution of 256x256 pixels.

(b) The CK database [51] includes 2105 digitized image sequences (video) from 182 adults ranging from 18 to 30 years old. Each image has a resolution of 640x490 pixels with eight-bit accuracy for gray scale values.

(c) The KDEF dataset [52] contains two sessions of multi-view posed facial expression images from 70 amateur actors, with age ranging from 20 to 30 years old. The database has totally $49002 \mathrm{D}$ images of seven human facial expressions of emotions. The images have a resolution of 562x762 pixels, and each of the seven facial expressions is acquired from five different angles $-90^{\circ},-45^{\circ}, 0^{\circ}, 45^{\circ}, 90^{\circ}$.

(d) The MUG Database [53] contains 86 subjects, where 51 are males and 35 are females. All subjects are between 20 and 35 years old. Only 52 subject images are available for usage with this database. For each expression, a total of 50 to 160 images are existing. The images have a resolution of 896x896 pixels.

\subsection{Results and analysis}

Tables 2 and 3 report the average accuracy of each tested descriptor obtained on CK, JAFFE, KDEF and MUG Databases. The first column consists of the name of the descriptor along with the parameter used if that concerns a parametric descriptor. The other columns concern the abbreviation of emotion categories that we tested and the accuracy obtained; NE: NEUTRAL, HA : HAPPY, FE : FEAR, SA: SAD, AN: ANGRY, DI: DISGUST, SU: SURPRISE, Acc: Accuracy.

\subsubsection{Performance analysis on Cohn-Kanade (CK) Database}

For this database, we used a subset of 10 sequences that reflect only the samples expressing the seven categories of emotions, and then we selected the four latest frames of each sequence that have the highest expression intensity. The optimal number of non-overlapping sub-regions to compute the histogram features is $14 \times 14$ for all the tested descriptors. For each emotion expression, two images are used as training set and the two others are used as test set. Table 2 illustrates the obtained experimental results for the basic emotion 
recognition recorded on $\mathrm{CK}$ dataset using the 46 evaluated state-of-the-art texture descriptors. It can be inferred that almost all the tested descriptors produce good results on CK dataset where their average accuracy is above 96\%. Tweenty-seven LBP-methods like RALBGC, BGC1, BGC2, BGC3, dLBP $\alpha$, ELGS manage successfully to differentiate all classes perfectly (average accuracy equal to 100\%), leaving then, essentially, no room for improvement. Note that, all the evaluated descriptors reached a score of $100 \%$ for "Happy" and "Surprise" classes.

\subsubsection{Performance analysis on JAFFE Database}

In this second experiment, each emotion in JAFFE database is designated into 10 females with three samples. One image is taken for each person and for each emotion expression in the test, making a total of 70 samples in the testing phase while the remaining 140 samples depict the training set. All faces are preprocessed to align them into a canonical images with a resolution of $128 \times 128$. The histograms are produced on the feature images spatially divided into $12 \times 12$ non-overlapping sub-regions. It is apparent from Table 2 that DSLGS, ELGS and SLGS operators yield the highest average rate as they reached a score of $98.57 \%$. Then, come the eight descriptors: BGC2, CSLBP, dLBP $\alpha$, ILBP, LCCMSP, LDENP, LGCP and OS_LTP which reached a recognition rate of $97.14 \%$. It can be noticed that several tested LBP-like descriptors have perfectly recognized some classes by getting the accuracy of $100 \%$.

Note that there is a significant performance drop for all the tested descriptors on the class of "sadness" where the reached accuracy is in the range [50\%, 90\%]. It also emerges from Table 2 that some methods like CSALTP, GTUC and LMEBP produce the worst performance on almost all the classes where their accuracy is sometime below $70 \%$. We would also point out that although parametric methods like eCS_LTP, ILTP, GTUC, AELTP are regarded as "optimized" since their parameter values are tuned during the experiment, their performance is markedly weaker than the non-parametric ones.

\subsubsection{Performance analysis on KDEF database}

We choose the images of both sessions for each subject and only the view angle $0^{\circ}$ is considered. The subset contains 70 subjects, each one expresses two times the seven emotion categories. Thus, in total we use 980 images. We altered the sizes of all the faces of KDEF database into a steady sized template, which have the same resolution of 256x256 and the faces were then split into $14 \times 14$ blocks for region-based feature extraction. Each subject express two times the seven categories, so we selected one facial image per subject for training phase and the other one for test phase.

It is apparent from Table 3 that the LGS operator is ranked as the top 1 descriptor in KDEF database as it achieves a recognition rate of $95.92 \%$, with perfect recognition (100\%) of happy and neutral categories, followed by DSLGS, SLGS and LBP descriptors which reached a score of $95.31 \%$. Then, come seven descriptors like BGC2, BGC3, CSLBP, dLBP $\alpha$, ELGS, ILBP and LQPAT which allowed to achieve accuracies between [94.08\% - 94.90\%]. Then tweenty-six LBP-methods attained accuracies between [90.20\% - 93.88\%] where three descriptors RLBP, BGC1 and SMEPOP reached 93.88\% and two descriptors MMEPOP and DBC attained $90.20 \%$ and $90.41 \%$, respectively. Accuracies between [80.61\% - 86.53\%] were achieved by eight LBP-like methods in which $80.61 \%$ was achieved by ALTP and $86.53 \%$ by XCS_LBP. We can observe from Table 3 that the worst performance of $59.39 \%$ was attained by CSALTP descriptor.

\subsubsection{Performance analysis on Multimedia Understanding Group (MUG) Database}

We have used 924 facial expression images, i.e., 132 images for each facial expression. All faces were altered and resized to have a common resolution of 256x256. Then, they were split into $18 \times 18$ blocks for region-based feature extraction. For this experiment, in each emotion category, we used four images per subject, two for training phase and two for test phase.

Table 3 gathers the obtained experimental results. Clearly, it can be observed that eight of the tested descriptors ELGS, LDTP, LDENP, LGCP, LNDP, LTP, LQPAT and SMEPOP manage to differentiate all classes perfectly $100 \%$ in accuracy leaving then, no room for improvement. In addition, thirty-one LBP-like methods give accuracies between [99.03\% - 99.68\%], LBP attained 98.73\%, DBC reached 98.05\%, XCS_LBP got $97.40 \%$ and finally, GTUC attained an accuracy of $97.08 \%$.

As we can observe, all tested methods obtain very promising results on the MUG dataset, excpect three state-of-the-art methods AELTP, LMEBP and CSALTP attained the lowest accuracies comparing with the other methods tested. The undermost accuracy of $71.43 \%$ was achieved by CSALTP. Then an accuracy of $84.09 \%$ was attained by AELTP and finally $89.94 \%$ was obtained when testing LMEBP method. 
Table 2. Experiments Results on CK and JAFFE Databases

\begin{tabular}{|c|c|c|c|c|c|c|c|c|c|c|c|c|c|c|c|c|}
\hline & \multicolumn{8}{|c|}{ Cohn Canade Database } & \multicolumn{8}{|c|}{ JAFFE Database } \\
\hline & $\mathrm{NE}$ & HA & FE & DI & $\mathrm{AN}$ & SA & SU & Acc & $\mathrm{NE}$ & HA & FE & DI & $\mathrm{AN}$ & SA & SU & Acc \\
\hline LDIr & 100 & 100 & 00 & 95 & 95 & 100 & 100 & 98.57 & 90 & 90 & 80 & 90 & 60 & 70 & 100 & 2.86 \\
\hline RALB & 00 & 100 & 100 & 100 & 100 & 100 & 100 & 100 & 90 & 100 & 80 & 90 & 80 & 80 & 100 & 38.57 \\
\hline RLBP & 100 & 100 & 00 & 100 & 100 & 100 & 100 & 100 & 90 & 90 & 90 & 80 & 90 & 80 & 100 & 38.57 \\
\hline CELDP & 100 & 100 & 100 & 100 & 100 & 100 & 100 & 100 & 90 & 80 & 80 & 80 & 100 & 80 & 100 & 7.14 \\
\hline & 95 & 100 & 100 & 100 & 95 & 95 & 100 & 97.86 & 80 & 80 & 90 & 80 & 90 & 80 & 100 & 5.71 \\
\hline $\operatorname{ALTP}\{0.006$ & s & 100 & 100 & 100 & 95 & 95 & 100 & 97.14 & 100 & 100 & 90 & 90 & 100 & 80 & 100 & 4.29 \\
\hline & & 100 & 100 & 100 & 100 & 100 & 100 & 100 & 90 & & 80 & 100 & 100 & 80 & 100 & 91.43 \\
\hline & & 100 & 100 & 100 & 100 & 100 & 100 & 100 & 100 & 100 & 100 & 100 & 100 & 80 & 100 & \\
\hline & & 100 & 100 & 100 & & & & & & & & & 100 & 80 & 100 & \\
\hline & & 100 & 100 & 90 & & 90 & & & 100 & & & & 100 & 90 & 100 & \\
\hline CSALTP $\{0.0$ & 100 & 100 & 100 & 100 & & 95 & & & 70 & & & & 50 & 60 & 00 & \\
\hline $\operatorname{CSLBP}\{1\}$ & 100 & 100 & 100 & 100 & 100 & 100 & 100 & 100 & 100 & 100 & 100 & 100 & 100 & 80 & 100 & 97.14 \\
\hline CCIT & 100 & 100 & 100 & 100 & & & 100 & 100 & 100 & & & 100 & 90 & 80 & 100 & 4.29 \\
\hline & & 100 & 100 & 100 & & & 100 & & & & & 90 & 100 & 80 & 100 & \\
\hline DE & & & 100 & 95 & 95 & & & & & & & & 90 & 90 & 100 & \\
\hline & & & 100 & 100 & 95 & & & & 90 & & 100 & 100 & 100 & 80 & 100 & 94.29 \\
\hline & 100 & 100 & 100 & 100 & 100 & 100 & 100 & 100 & 100 & 100 & 100 & 90 & 100 & 90 & 100 & 97.14 \\
\hline DSLGS & 100 & 100 & 100 & 100 & 100 & 100 & 100 & 100 & 100 & 100 & 100 & 100 & 100 & 90 & 100 & 98.57 \\
\hline & 100 & 100 & 100 & 90 & 95 & 100 & 100 & 97.86 & 100 & 100 & 80 & 90 & 90 & 90 & 100 & 92.86 \\
\hline & & 100 & 100 & 100 & & & & & & & 100 & 100 & 100 & 90 & 100 & \\
\hline & & 100 & 95 & 95 & & & & & & & 60 & & 80 & 50 & 0 & \\
\hline & & & 100 & 100 & & & & & & & & 90 & 90 & 70 & 100 & .71 \\
\hline & 100 & 100 & 100 & 100 & 100 & 20 & 100 & 100 & 100 & & 100 & 100 & 100 & 90 & 100 & 97.14 \\
\hline $\operatorname{ILTP}\{1\}$ & 05 & 100 & 100 & 100 & 95 & 95 & 100 & 97.86 & 90 & 100 & 80 & 90 & 80 & 80 & 100 & 88.57 \\
\hline & & 100 & 100 & 100 & & & 100 & & 100 & & & 90 & 90 & 80 & 00 & 94.29 \\
\hline & & 100 & 100 & 100 & & & & & & & & 100 & 100 & 80 & 100 & 2.86 \\
\hline & & & 95 & 90 & & & & & & & 100 & 100 & 100 & 90 & 100 & \\
\hline & & & 100 & 100 & & & & & & & & & 100 & 80 & 100 & \\
\hline & & & 100 & 100 & & & & & & & & & 100 & 80 & 100 & 97.14 \\
\hline & & 100 & 100 & 100 & 100 & 100 & 100 & 100 & 100 & & 90 & 100 & 100 & 80 & 100 & 95.71 \\
\hline & & 100 & 100 & 100 & 100 & 100 & 100 & & 100 & 100 & 90 & 100 & 100 & 70 & 100 & 94.29 \\
\hline & & & 100 & 100 & & & & & & & & & 100 & 80 & 100 & .86 \\
\hline & & & 100 & 100 & & & & & & & 100 & & 100 & 80 & 100 & 97.14 \\
\hline & & 100 & 100 & 100 & 100 & & 100 & & 100 & & 90 & 100 & 100 & 80 & 100 & 95.71 \\
\hline & & 100 & 100 & 90 & 95 & & 100 & & 60 & & & 90 & 50 & 60 & 80 & 71.43 \\
\hline & & & 100 & 100 & 95 & 100 & 100 & & 90 & & 100 & 100 & 100 & 80 & 100 & 95.71 \\
\hline $\operatorname{LTP}\{1\}$ & & 100 & 100 & 100 & 95 & 95 & 100 & 97.14 & 90 & 100 & 90 & 90 & 100 & 80 & 100 & 92.86 \\
\hline & & 100 & 100 & 100 & 100 & 100 & 100 & & & 100 & 100 & 100 & 100 & 80 & 100 & 95.71 \\
\hline & 100 & 100 & 100 & 100 & 100 & 100 & 100 & & 100 & 100 & 90 & 90 & 100 & 80 & 100 & 94.29 \\
\hline & 100 & 100 & 100 & 100 & 100 & 100 & 100 & 100 & 100 & 90 & 90 & 100 & 100 & 80 & 100 & 94.29 \\
\hline OS_LTP $\{1\}$ & 100 & 100 & 100 & 100 & 100 & 100 & 100 & 100 & 100 & 100 & 100 & 100 & 100 & 80 & 100 & 97.14 \\
\hline & 100 & 100 & 100 & 95 & 100 & 100 & 100 & 99.29 & 100 & 100 & 90 & 100 & 100 & 70 & 100 & 94.29 \\
\hline & & 100 & 100 & 100 & 100 & & & & 100 & & 100 & 100 & 100 & 90 & 100 & 98.57 \\
\hline & 100 & 100 & 100 & 100 & 100 & 100 & 100 & 100 & 90 & 100 & 100 & 90 & 100 & 80 & 100 & 94.29 \\
\hline CaTr & 100 & 100 & 100 & 95 & 100 & 100 & 100 & 99.29 & 100 & 100 & 80 & 100 & 100 & 70 & 100 & 92.86 \\
\hline XCS_LBP & 100 & 100 & 100 & 100 & 95 & 100 & 100 & 99.29 & 90 & 100 & 90 & 70 & 90 & 80 & 100 & 88.57 \\
\hline
\end{tabular}


Table 3. Experiments Results on KDEF and MUG Databases

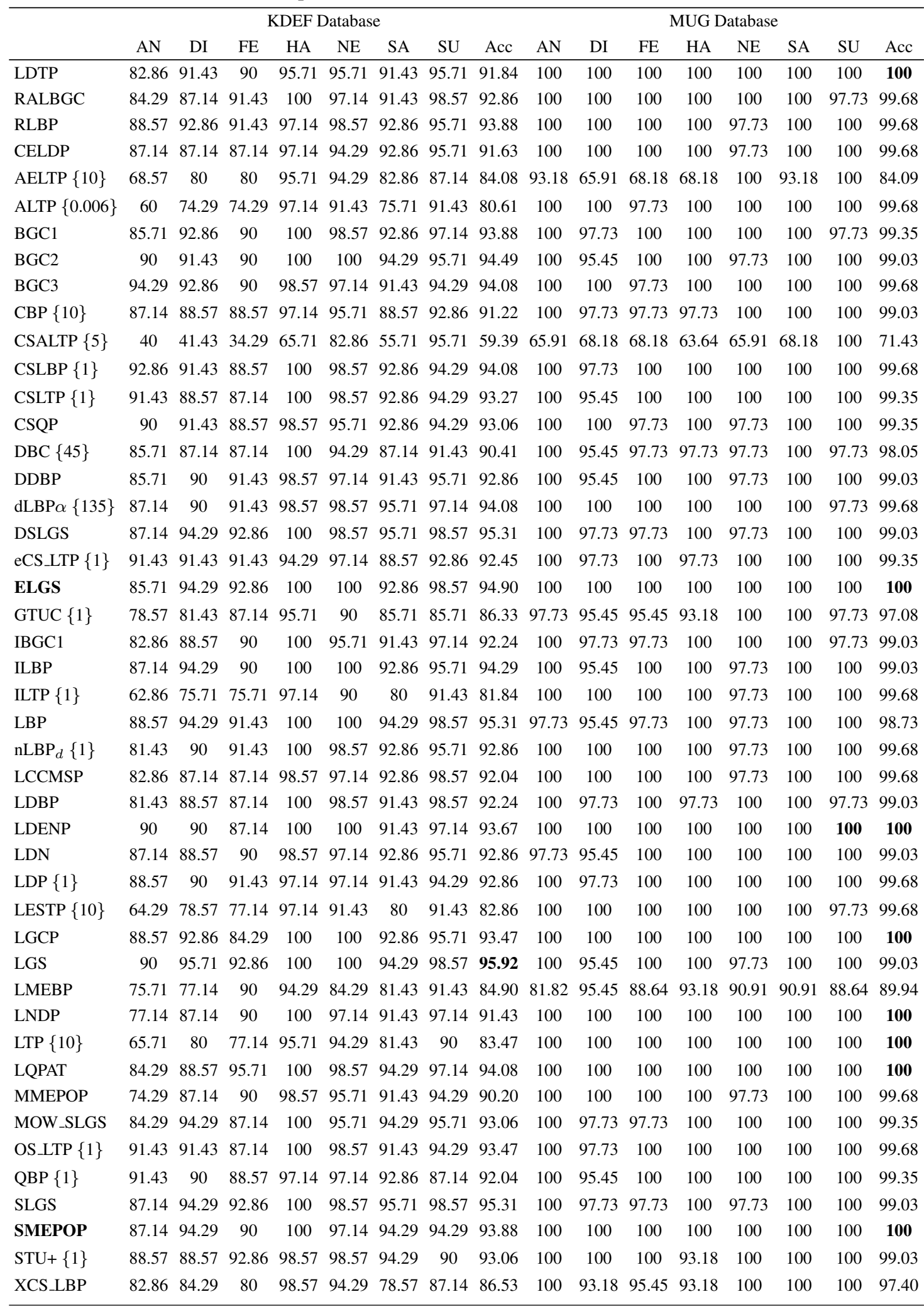




\subsection{Comparison with state-of-the-art methods}

In this section, we compare the performance of the best performing descriptors on each database with those of existing state-of-the-art methods. We should note that the performance evaluation with other stateof-the-art approaches may not be directly comparable due to the differences in partitioning the dataset into training and testing sets, number of classes, number of subjects and features used. However, distinctive results of every approach still can be indicated. The extracted results from the reviewed state-of-the-art papers as well as the recognition rates reached by the best performing evaluated LBP-variants on each database are arranged in Table 4.

It can be observed from Table 4 that, except for both JAFFE and KDEF databases, where the number of the used samples is relatively the same for almost all the existing systems, the used number of samples on $\mathrm{CK}$ and MUG databases varies from one existing approach to another. Given two different systems to compare on a given database, two cases are possible to provide a fair and accurate comparison of their results. In the first one, the used number of samples and the configuration into train/test sets should be the same, whereas in the second case, the system using a less number of samples, must at least be tested with a delicate configuration into train/test sets compared to the other which uses a higher number of samples. We used the second case in our evaluation for comparing the state-of-the-art methods with the adopted system, which uses the most difficult configuration in terms of train/test sets. Indeed, almost all the existing state-of-the-art systems use a partition where the number of training images is superior to that of test images (e.g., 10-fold), while in this study, the half-half configuration is adopted.

Table 4. Comparison with state-of-the-art methods

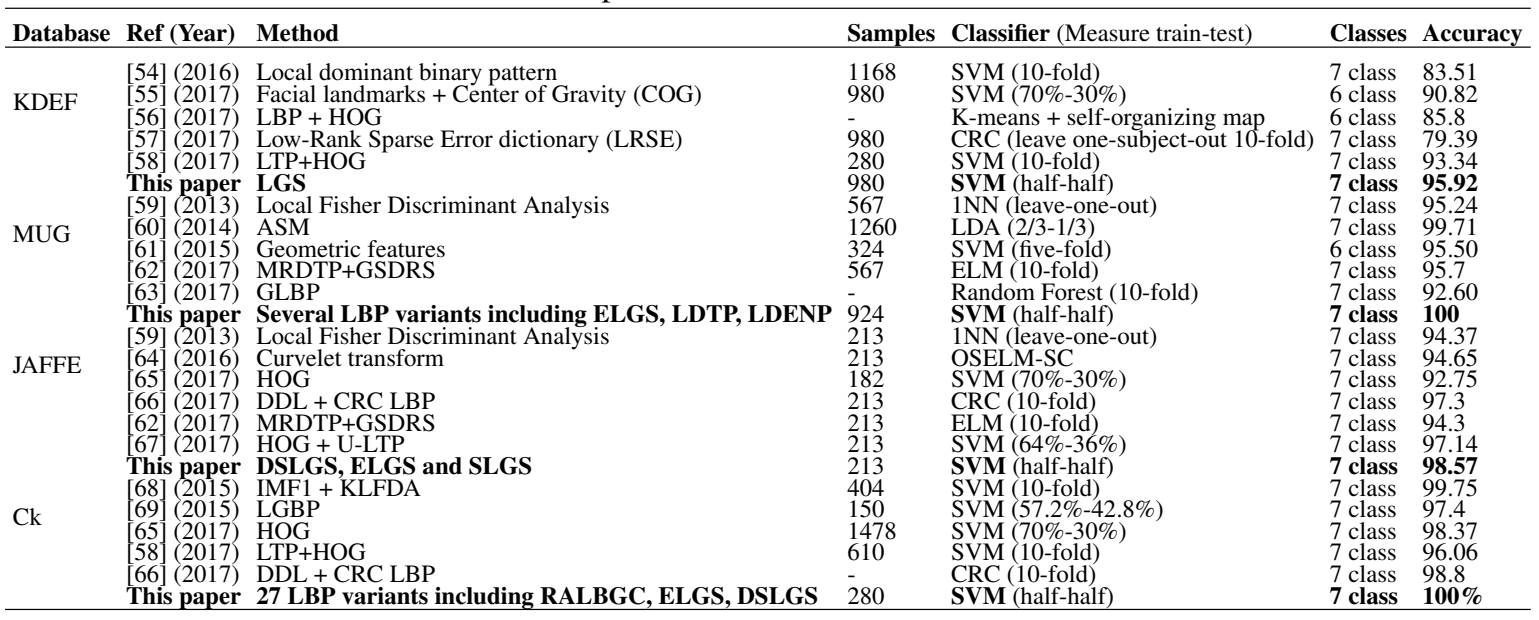

Examining Table 4, we could make the following findings :

(a) KDEF database: It can be easily observed that the LGS operator is the best performing method which achieved the higher performance over the recent state-of-the-art systems with a recognition rate reaching $95.92 \%$.

(b) JAFFE database: It is easily found that the accuracy recorded by three LBP-like variants outperformed those obtained by the state-of-the-art approaches. Indeed, it emerges from Table 4that the top ranked method on JAFFE database is that presented in [66] as it reached a score of $97.3 \%$ which is lower than that obtained by DSLGS, ELGS and SLGS operators (98.57\%).

(c) CK database: It is apparent from Table 4 that the highest score achieved on CK database is $99.75 \%$ obtained by the method presented in [68] while Table 2indicates that 27 LBP variants reached a score of $100 \%$.

(d) MUG database: As for CK database, several evaluated LBP variants like ELGS, LDTP, LDENP, LGCP, LNDP LQP and SMEPOP descriptors reached a score of $100 \%$ outperforming the best performing stateof-the-art approach presented in [60] which reached a score of $99.71 \%$.

The LGS, DSLGS and ELGS descriptors, which are based on the graph concept, manage to achieve remarkable accuracies over all the tested benchmarks. This fact is clearly highlighted on KDEF experiment where we find that few descriptors succeeded to record above $94 \%$ average accuracy. Then, the dominant graph 
encoding process justifies the robustness and effectiveness of LGS, DSLGS and ELGS descriptors. On the other hand, we remark that CSALTP descriptor suffers on KEDF experiment reaching just 59.39\% also on JAFFE and MUG experiments, on which the results were very high by the majority of the tested descriptors, the reason behind is the user specified threshold used in this operator, which needs to be identified on each experiment based on testing many values requiring many computations. Rather than this, all the other descriptors record good performances proving the discriminative power of the local description concept.

\section{CONCLUSION AND FUTURE WORKS}

We reported in this present work a comprehensive comparative experimental analysis of a great number of recent state-of-the-art LBP-like descriptors on facial expression recognition. It is noteworthy that the choice of an appropriate descriptor is crucial and generally depends on the intended application and many factors, such as computational efficiency, discriminative power, robustness to illumination and imaging system used. The experiments presented herein significantly constitute a good reference model when trying to find an appropriate method for a given application. Our experiments on facial expression recognition included a detailed and comprehensive performance study of 46 texture descriptors of the literature covering numerous application areas like texture classification, image retrieval, finger vein recognition, medical image analysis, face recognition, face expression analysis, etc. To show descriptors performance over several challenging situations, the tested descriptors were applied on four famous and widely used datasets such as JAFFE, CK, KDEF and MUG databases. The main finding that can be drawn from the analysis of the overall performance from the experiments is that although some LBP-like features have been originally conceived and proposed for texture classification, they show considerable performance in facial expression recognition. Indeed, even though they were not specifically designed for facial expression recognition, some LBP variants outperform all state-ofthe-art approaches over the tested databases. It is of great importance to note that the descriptors based on dominating set and graph present a significant performance stability against the other evaluated state-of-the-art descriptors as they are often found among the best performing LBP variants on the four tested databases. For KDEF database, LGS operator, which is based on dominating set and graph theory, is the best performing descriptor reaching a score of $95.92 \%$ outperforming the recent state-of-the-art systems. For JAFEE database, the better recognition rate which was $98.57 \%$ has been achieved by three descriptors based also on dominating set and graph theory such as DSLGS, ELGS and SLGS. 27 LBP variants including again those based on dominating set and graph theory reached a score of $100 \%$ on CK database. Finally, many evaluated LBP variants like LDTP, LDENP, LGCP, LNDP LQP and SMEPOP descriptors as well as the ELGS operator reached a score of $100 \%$ over MUG database. As future works, we look forward to extend this study to include the evaluation of deep features and deep classifiers. Furthermore, we wish to further explore the power of texture descriptors in other applications such as compound emotion recognition, gender classification, face recognition, texture classification, etc., in order to assess their ability to work with various classification problems.

\section{ACKNOWLEDGMENTS}

The authors are thankful to the National Center for Scientific and Technological Research for funding this research through the scholarship of excellence No 757UIT and No 7UIT2017 for the first and second authors. Our work was also part of the Volubilis AI 33/SI/14 program.

\section{REFERENCES}

[1] S.-J. Wang, W.-J. Yan et al., "Micro-expression recognition using robust principal component analysis and local spatiotemporal directional features," in Workshop at the European conference on computer vision. Springer, 2014, pp. 325-338.

[2] K. Slimani, R. Messoussi et al., "An intelligent system solution for improving the distance collaborative work," in Intelligent Systems and Computer Vision (ISCV). IEEE, 2017, pp. 1-4.

[3] S. Bourekkadi, S. Khoulji et al., "The design of a psychotherapy remote intelligent system," Journal of Theoretical and Applied Information Technology, vol. 93, no. 1, p. 116, 2016.

[4] T. Ojala, M. Pietikäinen et al., "Multiresolution gray-scale and rotation invariant texture classification with local binary patterns," IEEE Transactions on pattern analysis and machine intelligence, vol. 24, no. 7, pp. 971-987, 2002. 
[5] Y. Kaya, Ö. F. Ertuğrul et al., "Two novel local binary pattern descriptors for texture analysis," Applied Soft Computing, vol. 34, pp. 728-735, 2015.

[6] E. Abusham and H. Bashir, "Face recognition using local graph structure (lgs)," in International Conference on Human-Computer Interaction. Springer, 2011, pp. 169-175.

[7] H. K. Bashier, L. S. Hoe et al., "Texture classification via extended local graph structure," OptikInternational Journal for Light and Electron Optics, vol. 127, no. 2, pp. 638-643, 2016.

[8] M. Heikkilä, M. Pietikäinen et al., "Description of interest regions with center-symmetric local binary patterns," in ICVGIP, vol. 6. Springer, 2006, pp. 58-69.

[9] F. J. Madrid-Cuevas, R. M. Carnicer et al., "Simplified texture unit: a new descriptor of the local texture in gray-level images," Lecture notes in computer science, pp. 470-477, 2003.

[10] C.-I. Chang and Y. Chen, "Gradient texture unit coding for texture analysis," Optical Engineering, vol. 43, no. 8, pp. 1891-1903, 2004.

[11] S. Dong, J. Yang et al., "A new finger vein recognition method based on the difference symmetric local graph structure (dslgs)," International Journal of Signal Processing, Image Processing and Pattern Recognition, vol. 8, no. 10, pp. 71-80, 2015.

[12] X. Fu and W. Wei, "Centralized binary patterns embedded with image euclidean distance for facial expression recognition," in Fourth International Conference on Natural Computation, vol. 4. Springer, 2008, pp. 115-119.

[13] X. Tan and B. Triggs, "Enhanced local texture feature sets for face recognition under difficult lighting conditions," IEEE transactions on image processing, vol. 19, no. 6, pp. 1635-1650, 2010.

[14] B. Zhang, L. Zhang et al., "Directional binary code with application to polyu near-infrared face database," Pattern Recognition Letters, vol. 31, no. 14, pp. 2337-2344, 2010.

[15] L. Nanni, S. Brahnam et al., "A local approach based on a local binary patterns variant texture descriptor for classifying pain states," Expert Systems with Applications, vol. 37, no. 12, pp. 7888-7894, 2010.

[16] T. Jabid, M. Kabir et al., "Local directional pattern (ldp) for face recognition," in Digest of Technical Papers International Conference on Consumer Electronics (ICCE). IEEE, 2010, pp. 329-330.

[17] A. Fernández, M. X. Álvarez et al., "Image classification with binary gradient contours," Optics and Lasers in Engineering, vol. 49, no. 9, pp. 1177-1184, 2011.

[18] X. Wu and J. Sun, "An extended center-symmetric local ternary patterns for image retrieval," Advances in Computer Science, Environment, Ecoinformatics, and Education, pp. 359-364, 2011.

[19] J. Liu, X. Liu et al., "Improved local binary patterns for classification of masses using mammography," in International Conference on Systems, Man, and Cybernetics (SMC). IEEE, 2011, pp. 2692-2695.

[20] M. Subrahmanyam, R. Maheshwari et al., "Local maximum edge binary patterns: a new descriptor for image retrieval and object tracking," Signal Processing, vol. 92, no. 6, pp. 1467-1479, 2012.

[21] A. Rivera, J. Castillo et al., "Local directional number pattern for face analysis: Face and expression recognition," IEEE transactions on image processing, vol. 22, no. 5, pp. 1740-1752, 2013.

[22] M. Islam, "Local gray code pattern (lgcp): A robust feature descriptor for facial expression recognition," International Journal of Science and Research (IJSR), India Online ISSN, pp. 2319-7064, 2013.

[23] R. Mehta and K. O. Egiazarian, "Rotated local binary pattern (rlbp)-rotation invariant texture descriptor." in ICPRAM, 2013, pp. 497-502.

[24] K. Song, Y. Yan et al., "Adjacent evaluation of local binary pattern for texture classification," Journal of Visual Communication and Image Representation, vol. 33, pp. 323-339, 2015.

[25] S. Vipparth, S. Murala et al., "Local gabor maximum edge position octal patterns for image retrieval," Neurocomputing, vol. 167, pp. 336-345, 2015.

[26] S. Dong, J. Yang et al., "Finger vein recognition based on multi-orientation weighted symmetric local graph structure," KSII Transactions on Internet \& Information Systems, vol. 9, no. 10, 2015.

[27] M. Huang, Z. Mu et al., "Local image region description using orthogonal symmetric local ternary pattern," Pattern Recognition Letters, vol. 54, pp. 56-62, 2015.

[28] C. Silva, T. Bouwmans et al., "An extended center-symmetric local binary pattern for background modeling and subtraction in videos," in International Joint Conference on Computer Vision, Imaging and Computer Graphics Theory and Applications (VISAPP), 2015.

[29] W. Yang, Z. Wang et al., "Face recognition using adaptive local ternary patterns method," Neurocomputing, vol. 213, pp. 183-190, 2016.

[30] S. Rajput and J. Bharti, "A face recognition using linear-diagonal binary graph pattern feature extraction 
method," International Journal in Foundations of Computer Science \& Technology, vol. 6, no. 2, 2016.

[31] S. K. Vipparthi and S. K. Nagar, "Local extreme complete trio pattern for multimedia image retrieval system," International Journal of Automation and Computing, vol. 13, no. 5, pp. 457-467, 2016.

[32] H. Zeng, J. Chen et al., "Quad binary pattern and its application in mean-shift tracking," Neurocomputing, vol. 217, pp. 3-10, 2016.

[33] M. Faraji and X. Qi, "Face recognition under varying illuminations using logarithmic fractal dimensionbased complete eight local directional patterns," Neurocomputing, vol. 199, pp. 16-30, 2016.

[34] S. Chakraborty, S. Singh et al., "Centre symmetric quadruple pattern: A novel descriptor for facial image recognition and retrieval," Pattern Recognition Letters, 2017.

[35] P. S. Hiremath and R. A. Bhusnurmath, "Multiresolution ldbp descriptors for texture classification using anisotropic diffusion with an application to wood texture analysis," Pattern Recognition Letters, vol. 89, pp. 8-17, 2017.

[36] M. Verma and B. Raman, "Local neighborhood difference pattern: A new feature descriptor for natural and texture image retrieval," Multimedia Tools and Applications, pp. 1-24, 2017.

[37] S. Chakraborty, S. Singh et al., "Local quadruple pattern: A novel descriptor for facial image recognition and retrieval," Computers \& Electrical Engineering, vol. 62, pp. 92-104, 2017.

[38] A. Pillai, R. Soundrapandiyan et al., "Local diagonal extrema number pattern: A new feature descriptor for face recognition,” Future Generation Computer Systems, vol. 81, pp. 297-306, 2018.

[39] Y. El-merabet and R. Y, "Local concave-and-convex micro-structure patterns for texture classification," Pattern Recognition, vol. 76, pp. 303-322, 2018.

[40] I. Elkhadiri, A. Chahi et al., "Local directional ternary pattern: A new texture descriptor for texture classification," Computer Vision and Image Understanding, vol. 169, no. 14-27, 2018.

[41] I. Elkhadiri, M. Kas et al., "Repulsive-and-attractive local binary gradient contours: New and efficient feature descriptors for texture classification," Information Sciences, 2018.

[42] A. Hadid, J. Ylioinas et al., "Gender and texture classification: A comparative analysis using 13 variants of local binary patterns," Pattern Recognition Letters, vol. 68, pp. 231-238, 2015.

[43] A. Fernández, M. X. Álvarez et al., "Texture description through histograms of equivalent patterns," Journal of mathematical imaging and vision, vol. 45, no. 1, pp. 76-102, 2013.

[44] D. Huang, C. Shan et al., "Local binary patterns and its application to facial image analysis: a survey," IEEE Transactions on Systems, Man, and Cybernetics, Part C (Applications and Reviews), vol. 41, no. 6, pp. 765-781, 2011.

[45] L. Nanni, A. Lumini et al., "Survey on lbp based texture descriptors for image classification," Expert Systems with Applications, vol. 39, no. 3, pp. 3634-3641, 2012.

[46] M. Bereta, W. Pedrycz et al., "Local descriptors and similarity measures for frontal face recognition: a comparative analysis," Journal of visual communication and image representation, vol. 24, no. 8, pp. 1213-1231, 2013.

[47] A. Lumini, L. Nanni et al., "Ensemble of texture descriptors and classifiers for face recognition," Applied Computing and Informatics, vol. 13, no. 1, pp. 79-91, 2017.

[48] L. Liu, P. Fieguth et al., "Local binary features for texture classification: Taxonomy and experimental study," Pattern Recognition, vol. 62, pp. 135-160, 2017.

[49] K. Slimani, M. Kas et al., "Facial emotion recognition: A comparative analysis using 22 lbp variants," in Proceedings of the 2nd Mediterranean Conference on Pattern Recognition and Artificial Intelligence. ACM, 2018, pp. 88-94.

[50] M. Lyons, S. Akamatsu et al., "Coding facial expressions with gabor wavelets," in Proceedings of the third IEEE International Conference on Automatic Face and Gesture Recognition. IEEE, 1998, pp. 200-205.

[51] T. Kanade, J. Cohn et al., "Comprehensive database for facial expression analysis," in Proceedings of the Fourth IEEE International Conference on Automatic Face and Gesture Recognition. IEEE, 2000, pp. 46-53.

[52] M. Calvo and D. Lundqvist, "Facial expressions of emotion (kdef): Identification under different displayduration conditions," Behavior research methods, vol. 40, no. 1, pp. 109-115, 2008.

[53] N. Aifanti, C. Papachristou et al., "The mug facial expression database," in 11th International Workshop on Image Analysis for Multimedia Interactive Services (WIAMIS). IEEE, 2010, pp. 1-4.

[54] B. Santra and D. Mukherjee, "Local dominant binary patterns for recognition of multi-view facial ex- 
pressions," in Proceedings of the Tenth Indian Conference on Computer Vision, Graphics and Image Processing. ACM, 2016, p. 25.

[55] H. Alshamsi and V. Kupuska, "Real-time facial expression recognition app development on smart phones," Journal of Engineering Research and Application, vol. 7, pp. 30-38, 2017.

[56] S. A. Shah, G. Ali et al., "Cross-cultural emotion analysis a clustering approach," International Journal of Computer Science and Network Security, vol. 17, no. 8, pp. 35-41, 2017.

[57] Z. Sun, Z.-P. Hu et al., "Robust facial expression recognition with low-rank sparse error dictionary based probabilistic collaborative representation classification," International Journal on Artificial Intelligence Tools, vol. 26, no. 04, p. 1750017, 2017.

[58] K. Lekdioui, R. Messoussi et al., "Facial decomposition for expression recognition using texture/shape descriptors and svm classifier," Signal Processing: Image Communication, vol. 58, pp. 300-312, 2017.

[59] Y. Rahulamathavan, R.-W. Phan et al., "Facial expression recognition in the encrypted domain based on local fisher discriminant analysis," IEEE Transactions on Affective Computing, vol. 4, no. 1, pp. 83-92, 2013.

[60] C. Silva, A. Sobral et al., "An automatic facial expression recognition system evaluated by different classifiers," in X Workshop de Visao Computacinal (WVC 2014), Uberlandia, Minas Gerais, Brazil, 2014.

[61] D. Ghimire, J. Lee et al., "Recognition of facial expressions based on tracking and selection of discriminative geometric features," International Journal of Multimedia and Ubiquitous Engineering, vol. 10, no. 3, pp. 35-44, 2015.

[62] A. Alphonse and D. Dharma, "Novel directional patterns and a generalized supervised dimension reduction system (gsdrs) for facial emotion recognition," Multimedia Tools and Applications, pp. 1-34, 2017.

[63] M. A. Jaffar, "Facial expression recognition using hybrid texture features based ensemble classifier," International journal of advanced computer science and applications, vol. 8, no. 6, pp. 449-453, 2017.

[64] A. Uçar, Y. Demir et al., "A new facial expression recognition based on curvelet transform and online sequential extreme learning machine initialized with spherical clustering," Neural Computing and Applications, vol. 27, no. 1, pp. 131-142, 2016.

[65] U. Mlakar, I. Fister et al., "Multi-objective differential evolution for feature selection in facial expression recognition systems," Expert Systems with Applications, vol. 89, pp. 129-137, 2017.

[66] A. Moeini, K. Faez et al., "Facial expression recognition using dual dictionary learning," Journal of Visual Communication and Image Representation, vol. 45, pp. 20-33, 2017.

[67] M. Sajjad, A. Shah et al., "Facial appearance and texture feature-based robust facial expression recognition framework for sentiment knowledge discovery," Cluster Computing, pp. 1-19, 2017.

[68] H. Ali, M. Hariharan et al., "Facial emotion recognition using empirical mode decomposition," Expert Systems with Applications, vol. 42, no. 3, pp. 1261-1277, 2015.

[69] S. C. Neoh, L. Zhang et al., "Intelligent facial emotion recognition using a layered encoding cascade optimization model," Applied Soft Computing, vol. 34, pp. 72-93, 2015. 